\title{
Multilayer film applications in drug delivery: what's new and where to next?
}

\author{
"Commercializing the multilayer film technology is likely to involve \\ drug delivery. Vaccines seem a good bet..."
}

Keywords: encapsulation $\approx$ environmental responsiveness $\approx$ multilayer films $\approx$ polyelectrolytes $\approx$ vaccines

The multilayer films discussed in this editorial are prepared by an approach known as layerby-layer assembly. This coating method, which is a type of dip-coating procedure, was first described by Iler in the 1960s [1]. It was later developed by Decher and Lvov for oppositely charged polyelectrolytes and other charged molecules [2], and by Rubner and Yang [3] and Sukhishvili et al. [4] for polymer complexes stabilized by hydrogen bonds. Many different materials can be incorporated into multilayer films, as Iler noted. The research and commercialization effort of the present author on multilayer films has focused on one of the three classes of biological macromolecule, polypeptides [5]. These polymers can be purified from tissue, designed for film assembly or prepared by chemical means or genetic engineering of microorganisms, as in the pharmaceutical industry. In any case, particles are adsorbed onto a solid support under external control and the multilayer film is built up one 'layer' at a time. The approach owes its widespread popularity to its simplicity, affordability, automatability, scalability and versatility.

Many different applications of multilayer films have been suggested and a variety of applications have, indeed, been realized. Examples of potential and actual applications of these films include electrode and food coatings, as well as coatings for cell and tissue culture, and artificial red blood cells. Further remarks in the present work will be focused on drug-delivery applications. Technological purposes of multilayer films in this field include surface modification to improve drug solubility in an aqueous environment [6], encapsulation to protect biologics from the surrounding environment [7] and specific targeting to enhance drug efficacy or reduce side effects [8]. The films could also be utilized for drug reformulation; for example, to extend patent lifetime, which is a major interest of pharmaceutical companies today.

The majority of multilayer films are made of polymers that, in most cases, are oppositely charged polyelectrolytes. The film is built up by polymer adsorption onto a solid support. The substrates for film assembly in a drugdelivery context have included small crystals of hydrophobic drugs [6] and hydrogels impregnated with small-molecule drugs [9]. In such cases, the encapsulated substrate or material adsorbed onto the substrate plays the role of drug-delivery depot. Other approaches have been to make multilayer films from polymers onto which peptide drugs have been grafted [10], polymers from which drugs could be released by a change of environment [11] and polymers that incorporate T-cell antigens [12]. A common goal has been to fabricate films so as to make porosity, and therefore the rate of release of encapsulated materials, responsive to the surrounding environment.

The film-assembly process can be described as follows. A polycation is adsorbed onto a polyanionic surface, generally until binding sites are saturated. For films made of polyelectrolytes, electrostatic interactions both drive and limit polymer adsorption. After the substrate is rinsed to remove loosely bound material, a polyanion is adsorbed. In the process, soluble complexes are formed between oppositely charged polyelectrolytes on the film surface and the sign of charge of the film surface is reversed. Vodouhê et al., for example, have studied multilayer films made of poly(lysine), a polycation, and hyaluronic acid, a polyanion [13]. The investigators sought to characterize the accessibility of the antiproliferative agent paclitaxel $\left(\operatorname{Taxol}^{\circledR}\right)$ on the surface of the film in the absence of chemical modification of the polymers or the drug. The dose available to

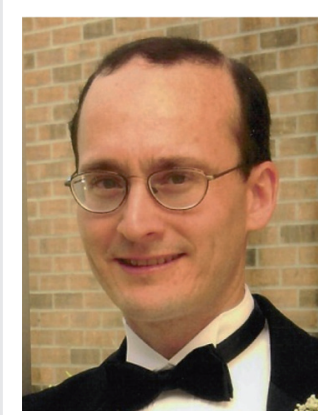

Donald T Haynie

University of South Florida,

Tampa, FL, USA

Tel.: + I 8139747793

Fax: +1 8139745813

E-mail: dhaynie@usf.edu 
cells seeded onto films in vitro was 'tuned' by controlling the number of 'inert' polyelectrolyte layers between the drug (inside the film) and the cells (outside the film). In such cases, the film acts as a diffusive barrier. The film may also protect encapsulated material from the surrounding environment or elements of the surrounding environment from encapsulated material.

The film assembly process need not involve oppositely charged polymers. In a study by Kim et al., for example, films were made of polycations and micelles with a negative surface charge [14]. The micelles were preloaded with a model drug and delivery was achieved by release of the drug from the micelles. Dendrimers and other materials have been utilized to reverse film-surface charge in place of a species of polyelectrolyte [15].

\section{"The films could also be utilized for drug reformulation; for example, to extend patent lifetime, which is a major interest of pharmaceutical companies today."}

Films made of polymers that interact by hydrogen bonds are relatively unstable. Hydrogen bonds are noncovalent interactions. Bond energies are comparable to thermal energy. Individual hydrogen bonds are 'weak', but collectively they can be thermostable. The relative instability of hydrogen bond networks presents possible advantages for drug delivery, as thermal fluctuations can be used to drive drug encapsulation and release. As mentioned above, Rubner and Sukhishvili carried out early work in this area [3,4].

In 2004, the stabilization of polyelectrolyte multilayer films by disulfide bonds was demonstrated [16]. The energy of a disulfide bond, which forms between two thiol groups, is comparable to that of a carbon-carbon bond. A crucial difference is the sensitivity of disulfides to the reducing potential of the surrounding environment. The polymers in this study were cysteine-containing polypeptides; cysteine, one of the 20 usual amino acids, has a thiol group in its side chain. It was then noted, in the context of microparticle encapsulation by multilayer films for possible drug-delivery applications that, whereas the external environment of cells is oxidizing and disulfide stabilizing, the internal environment is reducing and disulfide destabilizing [17]. In other words, the interior environment of a cell could be utilized to remove the coating from a capsule. Such capsules could then be taken up by cells by functionalizing the capsule surface, as others had demonstrated. Caruso and others have extended the basic concepts by attaching thiol groups to non-polypeptides that are used to build up multilayer films by hydrogen bond interactions [18] or using different polymers [19].

A current active area of research within the drug-delivery segment of the multilayer film community is vaccine development. Typical differences between investigators are the polymers selected for film fabrication, the means selected for film functionalization and the target indications. The utilization of coated microparticles or capsules for immunization holds the promise of the same particles serving both as immunogens and adjuvants. This topic and other relevant ones are covered in the recent review article by De Koker et al. [20].

A number of companies are actively engaged in commercialization activity involving multilayer films made of polymers. Capsulution, located in Berlin, Germany, is developing advanced drug-delivery carriers, medical devices and diagnostic particles. Their business model involves preparation of customized materials. Various synthetic organic polymers are utilized. Artificial Cell Technologies, Inc., (Science Park at Yale, New Haven, CT, USA) is developing synthetic vaccines. Microparticle coatings are designed to immunize patients against respiratory syncytial virus and the malaria parasite.

\section{"A current active area of research within the drug-delivery segment of the multilayer film community is vaccine development."}

Commercializing the multilayer film technology is likely to involve drug delivery. Vaccines seem a good bet, but other paths could lead to actual products. A device for transdermal delivery from a multilayer filmcoated material, for instance, would be easier to pass through a regulatory approval process than a vaccine formulation. The lower the likelihood of global impact and significant financial gain, however, the harder it will be to attract potential investors with deep pockets, industrial partners of size or buyers wellpositioned for manufacturing, packing and distribution throughout the world. 


\section{Financial \& competing interests disclosure}

The Editorial is written from an inventor's perspective. The author is a full-time employee of the University of South Florida (Tampa, FL, USA). The author has a financial interest in Artificial Cell Technologies, Inc. (New Haven, CT, USA). The author has no other relevant affliations or financial involvement with any organization or entity with a financial interest in or financial conflict with the subject matter or materials discussed in the manuscript apart from those disclosed.

No writing assistance was utilized in the production of this manuscript.

\section{References}

1 Iler RK. Multilayers of colloidal particles. J. Colloid Interf. Sci. 21, 569-594 (1966).

2 Lvov Y, Decher G, Möhwald H. Assembly, structural characterization and thermal behavior of layer-by-layer deposited ultrathin. Langmuir 9, 481-486 (1993).

3 Yang SY, Rubner MF. Micropatterning of polymer thin films using $\mathrm{pH}$-sensitive and crosslinkable polyelectrolyte multilayers. J. Am. Chem. Soc. 124, 2100-2101 (2002).

4 Kozlovskaya V, Ok S, Sousa A, Libera M, Sukhishvili SA. Hydrogen-bonded capsules formed by layer-by-layer self-assembly. Macromolecules 36, 8590-8592 (2003).

5 Haynie DT, Zhang L, Rudra JS, Zhao W, Zhong Y, Palath N. Polypeptide multilayer films. Biomacromolecules 6, 2895-2913 (2005).
6 Agarwal A, Lvov Y, Sawant R, Torchilin V. Converting poorly soluble materials into stable aqueous nanocolloids. J. Control. Release 128, 255-260 (2008).

7 Lvov Y, Antipov AA, Mamedov A, Möhwald $H$, Sukhorukov GB. Urease encapsulation in nanoorganized microshells. Nano Lett. 1, 125-128 (2001).

8 Matsusaki M, Akashi M. Functional multilayered capsules for targeting and local drug delivery. Exp. Op. Drug Del. 6, 1207-1217 (2009).

9 Su X, Kim B-S, Kim SR, Hammond PT, Irvine DJ. Layer-by-layer-assembled multilayer films for transcutaneous drug and vaccine delivery. ACS Nano 3, 3719-3729 (2009).

10 Chluba J, Voegel JC, Decher G, Erbacher P, Schaaf P, Ogier J. Peptide hormone covalently bound to polyelectrolytes and embedded into multilayer architectures conserving full biological activity. Biomacromolecules 2, 800-805 (2001).

11 Zhong Y, Whittington CF, Haynie DT. Stimulated release of small molecules from polyelectrolyte multilayer nanocoatings. Chem. Commun. 1415-1417 (2007).

12 Powell TJ, Palath N, DeRome ME, Tang J, Jacobs A, Boyd JG. Synthetic nanoparticle vaccines produced by layer-by-layer assembly of artificial biofilms induce potent protective T-cell and antibody responses in vivo. Vaccine 29, 558-569 (2011).

13 Vodouhê C, Guen EL, Garza JM et al. Control of drug accessibility on functional polyelectrolyte multilayer films. Biomaterials 27, 4149-4156 (2006).

14 Kim B-S, Lee H, Min Y, Poon Z, Hammond PT. Hydrogen-bonded multilayer of $\mathrm{pH}$-responsive polymeric micelles with tannic acid for surface drug delivery. Chem. Comm. 4194-4196 (2009).

15 Khopade AJ, Caruso F. Electrostatically assembled polyelectrolyte/dendrimer multilayer film as ultrathin nanoreservoirs. Nano Lett. 2, 415-418 (2002).

16 Li B, Haynie DT. Multilayer biomimetics: reversible covalent stabilization of a nanostructured biofilm. Biomacromolecules 5 , 1667-1670 (2004).

17 Haynie DT, Palath N, Liu Y, Li B, Pargaonkar, N. Biomimetic nanotechnology: inherent reversible stabilization of polypeptide microcapsules. Langmuir 21, 1136-1138 (2005).

18 Zelikin AN, Quinn JF, Caruso F. Disulfide cross-linked polymer capsules: en route to biodeconstructible systems. Biomacromolecules 7, 27-30 (2006).

19 Ma YJ, Dong MF, Hempenius MA, Möhwald H, Vancso GJ. Redox-controlled molecular permeability of composite-wall microcapsules. Nat. Mater. 5, 724-729 (2006).

20 De Koker S, Hoogenboom R, De Geest BG. Polymer multilayer capsules for drug delivery. Chem. Soc. Rev. 41, 2867-2884 (2012). 